\title{
Auf dem neuesten Stand? - Allergische Dermatitis bei der Katze
}

Ariane Neuber Watts

Allergische Dermatitiden sind ein multifaktorielles Krankheitsgeschehen. Nur eine Kombination verschiedener Therapieansätze ermöglicht der Katze eine gute Lebensqualität. Dabei gilt es, Stress zu minimieren sowie kausale und symptomatische Therapien zu kombinieren.

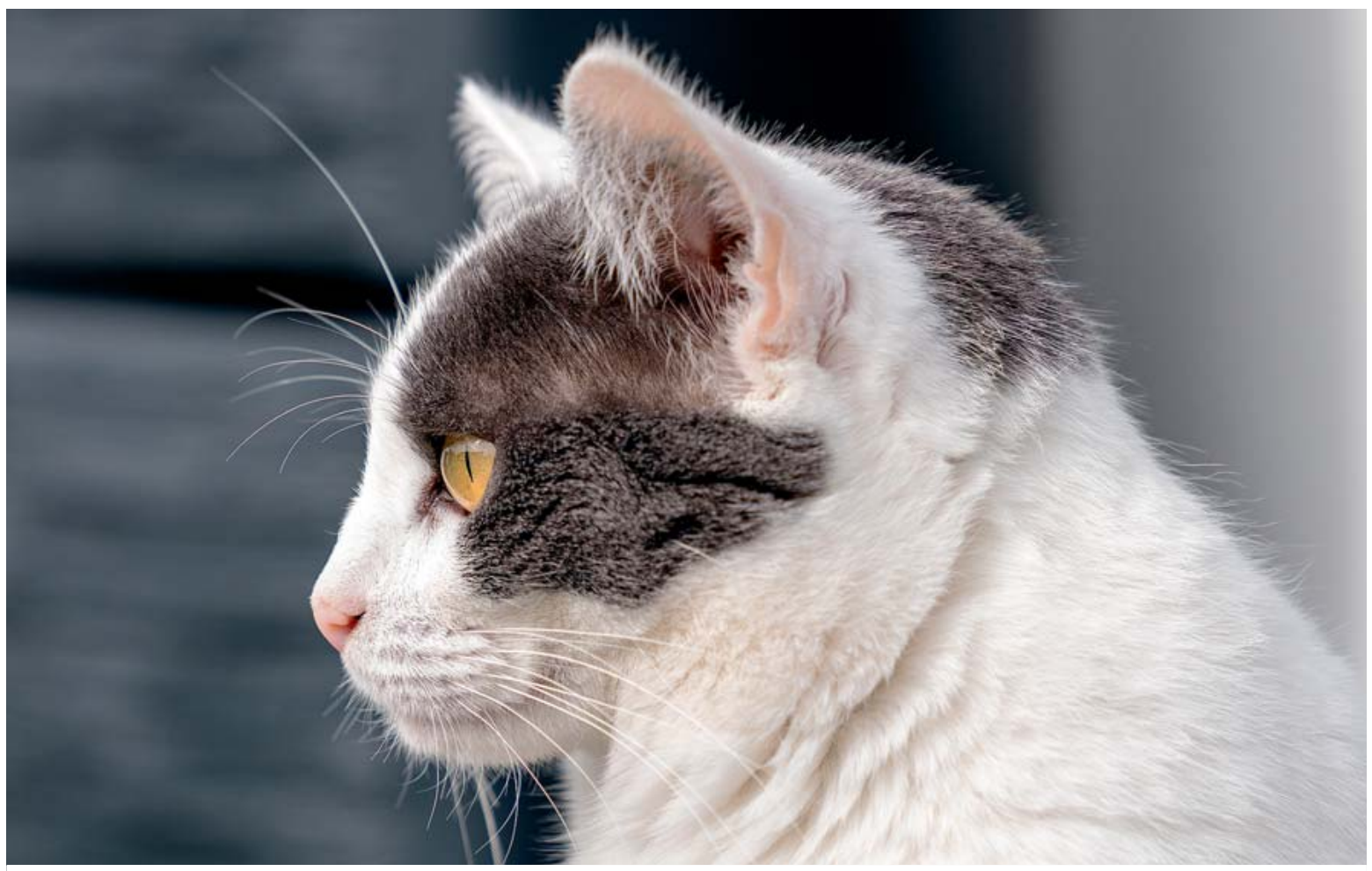

Quelle: Kirsten Oborny

\section{Welche diagnostischen Möglichkeiten gibt es?}

Beim Hund ist die atopische Dermatitis definiert als eine genetisch prädisponierte inflammatorische und juckreizauslösende allergische Hauterkrankung mit charakteristischen Eigenschaften, die meist mit allergenspezifischem IgE einhergeht [1, 2]. Diese Definition dient als Grundlage für die Diagnose der caninen atopischen Dermatitis
(CAD). In Fällen, bei denen die gleichen klinischen Eigenschaften, aber kein messbares Allergen-spezifisches IgE vorliegt, wird die Erkrankung als Atopie-ähnliche Dermatitis bezeichnet.

Diese Definition ist bei der Katze so nicht haltbar:

- Bei Katzen gibt es kaum überzeugende Beweise für eine genetische Prädisposition bestimmter Katzenrassen oder Individuen. 


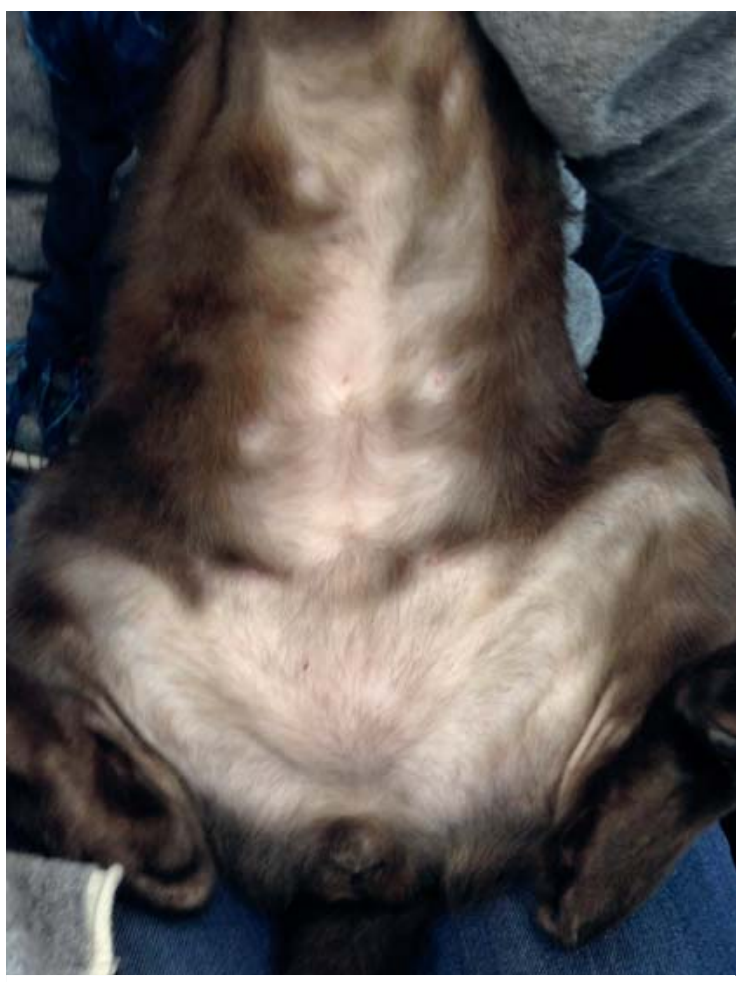

- Abb. 1 Katze mit selbstinduzierter Alopezie am ventralen Abdomen aufgrund einer Umweltallergie. Quelle: Ariane Neuber Watts

- Auch bei Katzen ist die Erkrankung pruritisch, allerdings gibt es keinen spezifischen Phenotyp, also keine charakteristischen klinischen Symptome.

- Die Patienten werden stattdessen mit einem der felinen kutanen Reaktionsmuster vorgestellt:

- symmetrische Alopezie ( $\bullet$ Abb. 1, miliare Dermatitis, Kopf- und Hals-Juckreiz ( Abb. 2, Abb. 3)

- eosinophiles Granulom-Komplex ( $\mathbf{A b b} \mathbf{4}$ )

- Diese Reaktionsmuster sind allerdings nicht spezifisch für Katzen mit einer Allergie, sondern können auch von anderen Erkrankungen ausgelöst werden.

- Die Rolle für Allergen-spezifisches IgE ist bei Katzen umstrittener als beim Hund.

Favrot et al. [3] haben diagnostische Kriterien für Katzen mit einer nicht Floh-induzierten Hypersensitivitätsdermatitis (nach Ausschluss von Flöhen) wie folgt beschrieben:

- Pruritus zu Beginn der Erkrankung

- Präsenz von mindestens 2 der folgenden klinischen Reaktionsmuster:

- symmetrische Alopezie

- miliare Dermatitis

- eosinophile Dermatitis

- Kopf- und Halserosionen/-ulzerationen

- mindestens 2 Körperstellen sind involviert

- miliare Dermatitis ist ein dominantes Reaktionsmuster

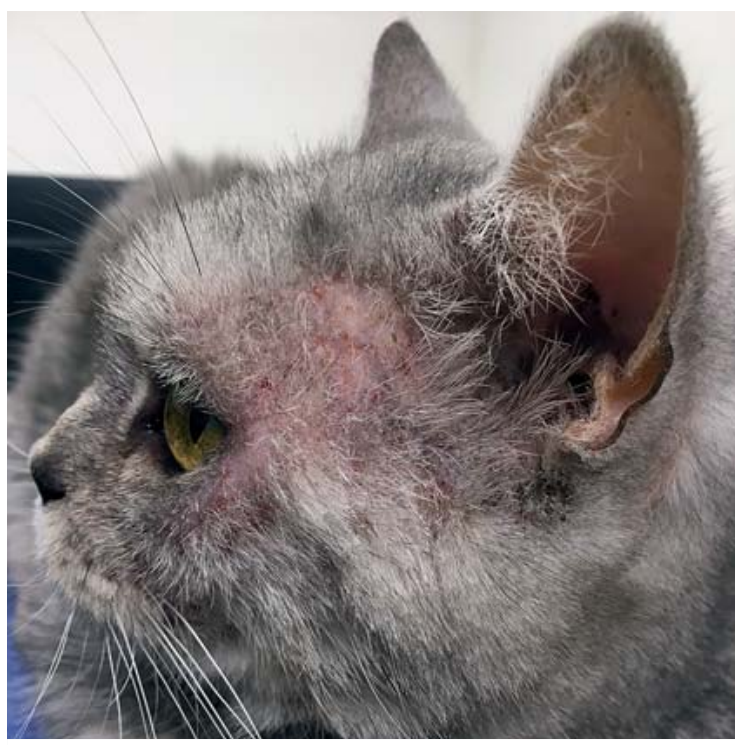

- Abb. 2 Katze mit Läsionen im präauricularen Bereich mit starkem Kopf- und Halspruritus aufgrund einer allergischen Dermatitis. Quelle: Ariane Neuber Watts

- Präsenz von eosinophiler Dermatitis oder symmetrischer Alopezie oder Erosionen/Ulzerationen an Gesicht, Lippen, Ohren oder Hals

- Präsenz von nicht symmetrischer Alopezie an Rumpf, Schwanz oder Hintergliedmaßen

- Präsenz von symmetrischer Alopezie des Abdomens

- Nichtvorhandensein von Erosionen/Ulzerationen der Vordergliedmaßen

- Nichtvorhandensein von sternalen und axillaren Läsionen

- Nichtvorhandensein von Knoten oder Tumoren

Werden 6 dieser 10 Kriterien erfüllt, ergibt sich eine Sensitivität von $90 \%$ und eine Spezifizität von $83 \%$ für die Diagnose nicht Floh-induzierte Hypersensitivitätsdermatitis.

Hobi et al. [4] beschrieben klinische Charakteristika von Katzen mit Hypersensitivitätsdermatitiden. Sie untersuchten die Häufigkeit von Symptomen bezogen auf die Körperregion. Die Beteiligung eines Körperteils im Gegensatz zu einem anderen schließt Flöhe, Futter und Umweltallergene nicht als kausale Faktoren aus. Allerdings fanden sie heraus, dass Futter-induzierte Hypersensitivität bei der Katze häufiger Läsionen im Kopf- und Halsbereich verursacht und häufiger mit Läsionen am kaudalen Dorsum vergesellschaftet ist ( $\triangleright$ Abb. 5). Trotzdem gibt es eine große Überschneidung von Symptomen von Flohbissallergie ( $\triangleright$ Abb.6), Futtermittel-induzierter Hypersensitivitätsdermatitis und nicht Floh-, nicht Futtermittel-induzierter Hypersensitivitätsdermatitis bei der Katze. Es bleibt, analog zur Situation beim Hund, eine Ausschlussdiagnose. 

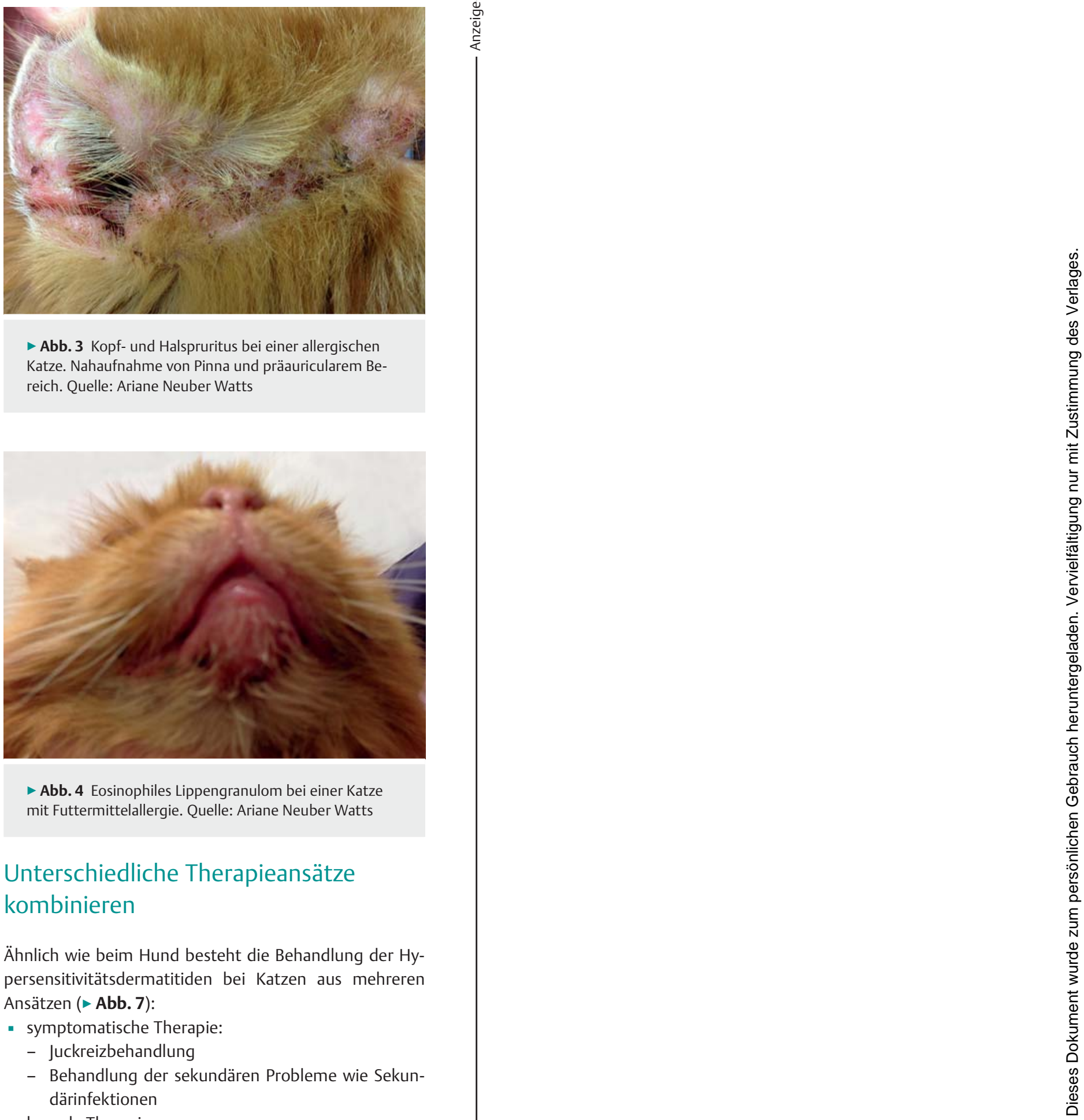

- Abb. 3 Kopf- und Halspruritus bei einer allergischen Katze. Nahaufnahme von Pinna und präauricularem Bereich. Quelle: Ariane Neuber Watts

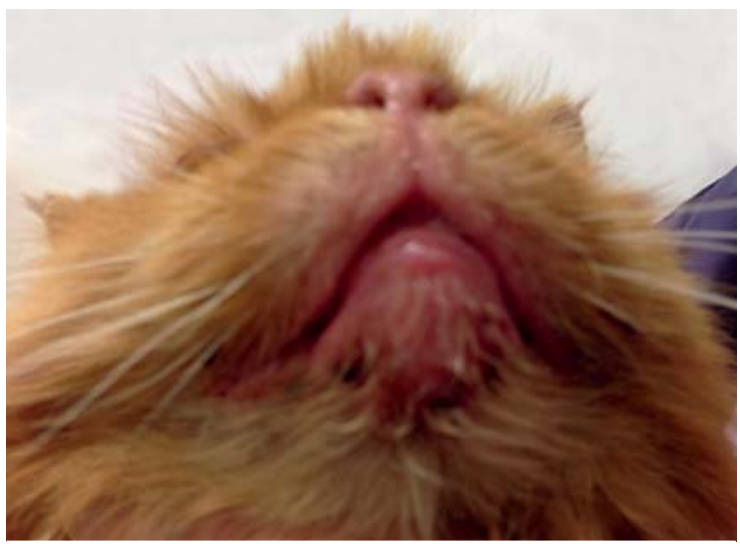

- Abb. 4 Eosinophiles Lippengranulom bei einer Katze mit Futtermittelallergie. Quelle: Ariane Neuber Watts

\section{Unterschiedliche Therapieansätze} kombinieren

Ähnlich wie beim Hund besteht die Behandlung der Hypersensitivitätsdermatitiden bei Katzen aus mehreren Ansätzen ( $\triangleright$ Abb. 7):

- symptomatische Therapie:

- Juckreizbehandlung

- Behandlung der sekundären Probleme wie Sekundärinfektionen

- kausale Therapie:

- häufig Allergen-spezifische Immuntherapie

- selten Allergenvermeidung

\section{Schwellenwert und}

\section{Verschlimmerungsfaktoren beachten}

Allergische Dermatitiden stellen ein multifaktorielles Geschehen dar. Etliche Katzen reagieren nicht nur auf eine Allergengruppe (Flöhe/andere Parasiten, Futter, Umwelt), sondern sind auf mehrere Allergengruppen sensitiviert. 


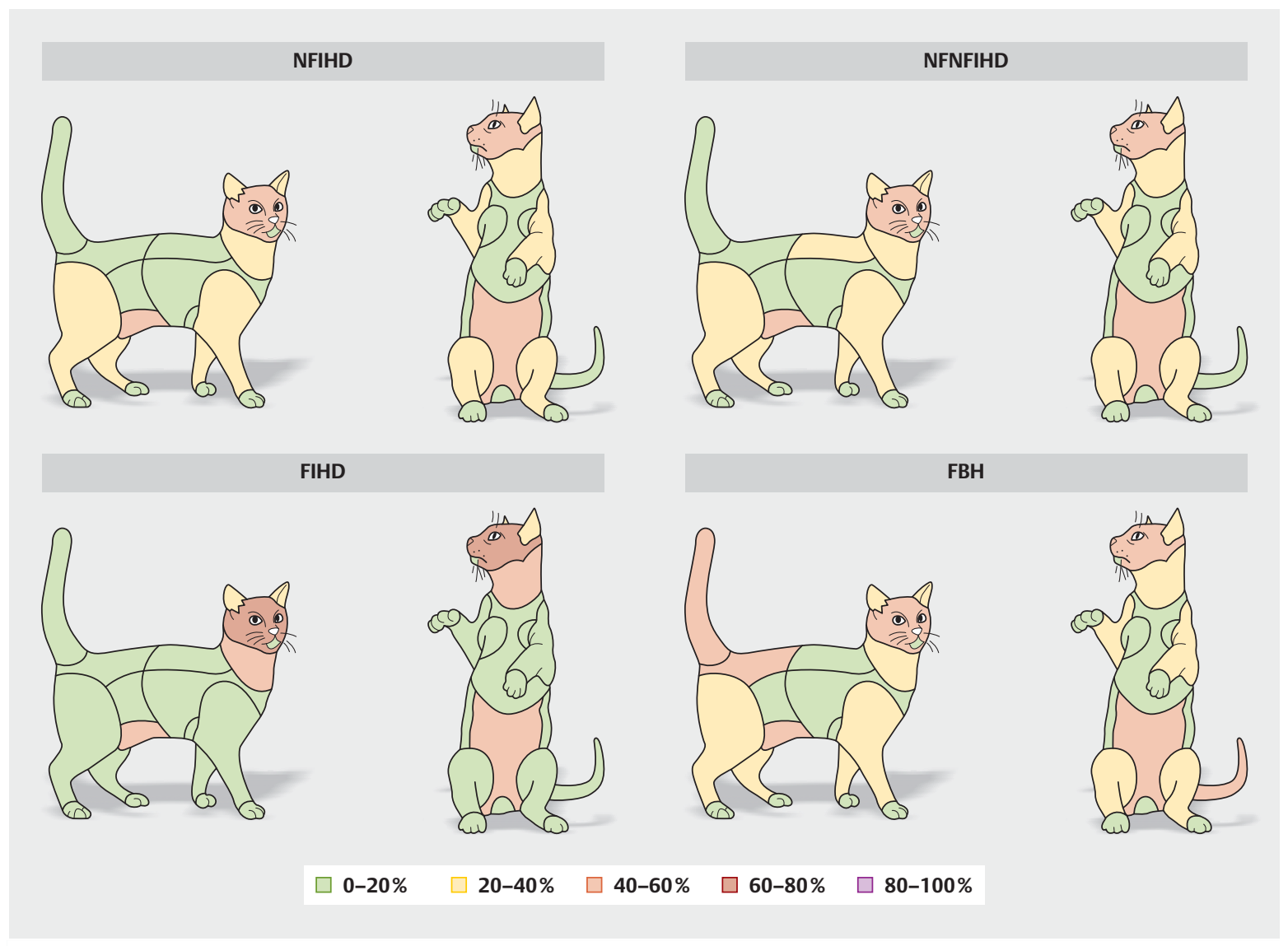

- Abb. 5 Silhouetten, die die Proportion von Läsionsverteilungsmustern bei Katzen mit nicht Floh-, nicht Futtermittel-induzierter und Flohbiss-Hypersensitivität darstellen: NFIHD = nicht Futter-induzierte Hypersensitivitätsdermatitis, NFNFIHD = nicht Floh-, nicht Futter-induzierte Hypersensitivitätsdermatitis, FIHD = Futter-induzierte Hypersensitivitätsdermatitis, FBH = Flohbiss-Hypersensitivitätsdermatitis. Quelle: Thieme Gruppe nach einer Vorlage von Hobi et al. [4].

Eine Reihe von Faktoren komplizieren und verschlimmern auch bei der Katze die Grunderkrankung Allergie. Sie stellen eine echte Herausforderung dar.

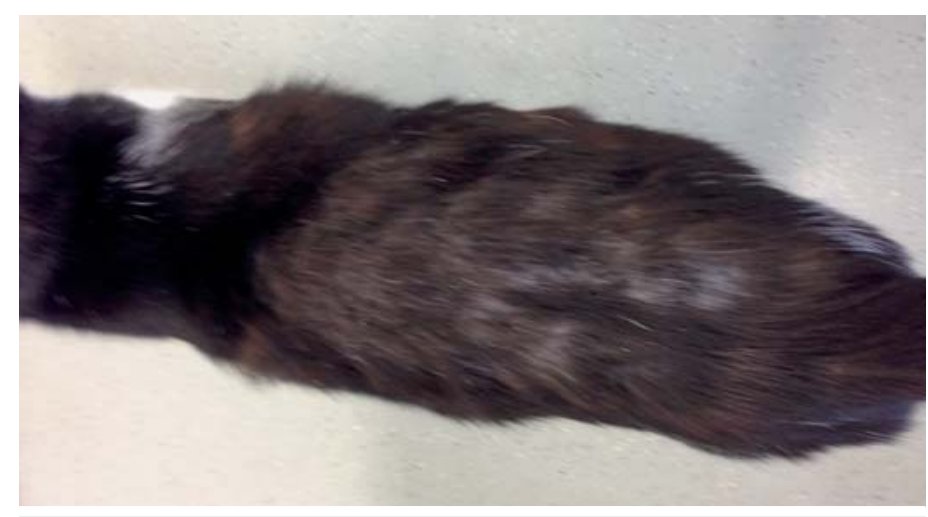

- Abb. 6 Katze mit Flohbiss-Hypersensitivitätsdermatitis mit kaudodorsaler Alopezie. Quelle: Ariane Neuber Watts

\author{
Merke \\ Je mehr dieser individuellen Faktoren erfolgreich \\ kontrolliert werden, desto erfolgreicher ist die Be- \\ handlung. \\ Mögliche Verschlimmerungsfaktoren bei felinen Allergi- \\ kern sind: \\ - Verhaltensprobleme/Stress \\ - Sekundärinfektionen mit Bakterien oder Hefen \\ - Ektoparasitenbefall (auch ohne Flohbissallergie, \\ - Abb. 8, Abb. 9) \\ - trockene Haut \\ - Kontaktallergie/-irritation \\ - Wärme \\ - Feuchtigkeit
}

Jedem dieser Faktoren kann man fiktive „Juckreizpunkte“ zuordnen, um grafisch das Schwellenwertprinzip ( $\triangleright$ Abb. 10) zu erklären. Stress spielt bei der Katze oft eine große Rolle, vor allem in Haushalten mit mehreren Katzen. 


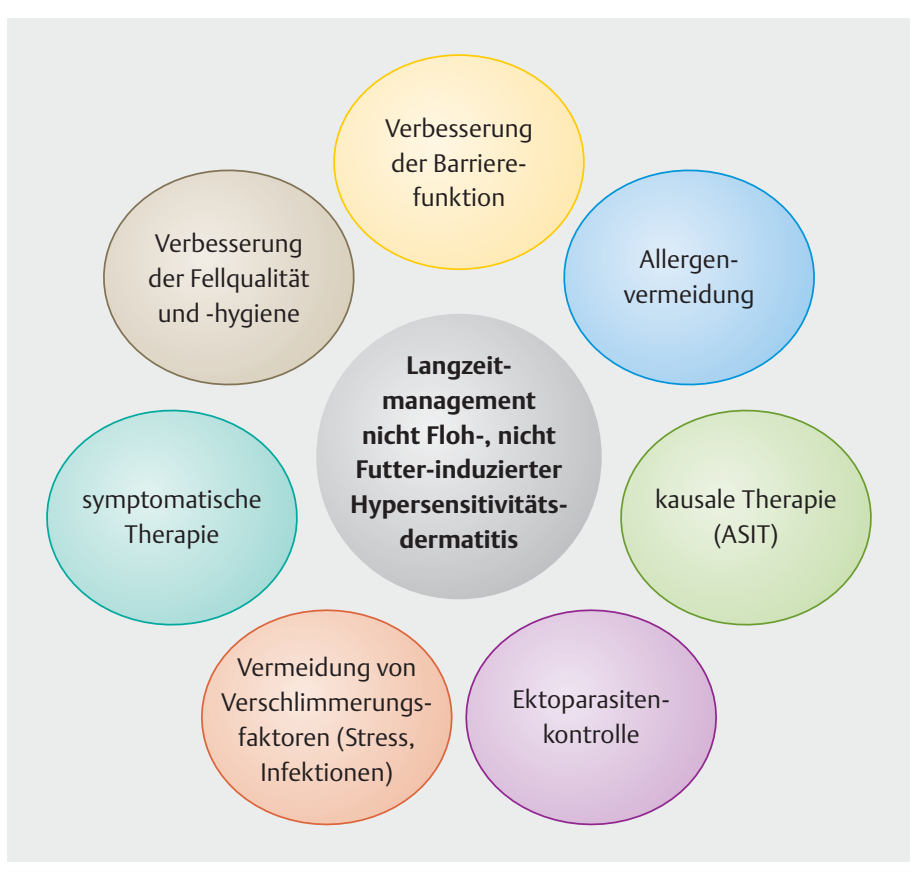

- Abb. 7 Faktoren, die bei der Behandlung von Katzen mit Umweltallergie beachtet werden sollten. Quelle: Thieme Gruppe nach einer Vorlage von Ariane Neuber Watts

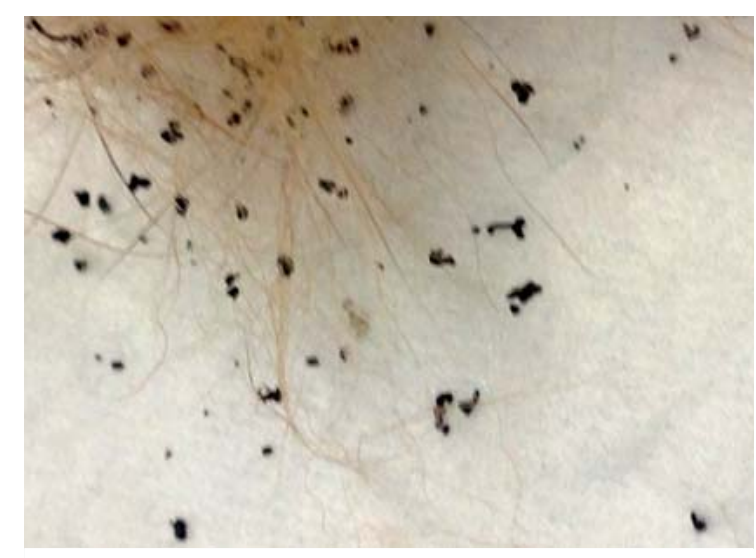

- Abb. 8 Flohkot auf weißem Papier durch Kämmen mit Flohkamm und Befeuchten des Papiers nachgewiesen. Quelle: Ariane Neuber Watts

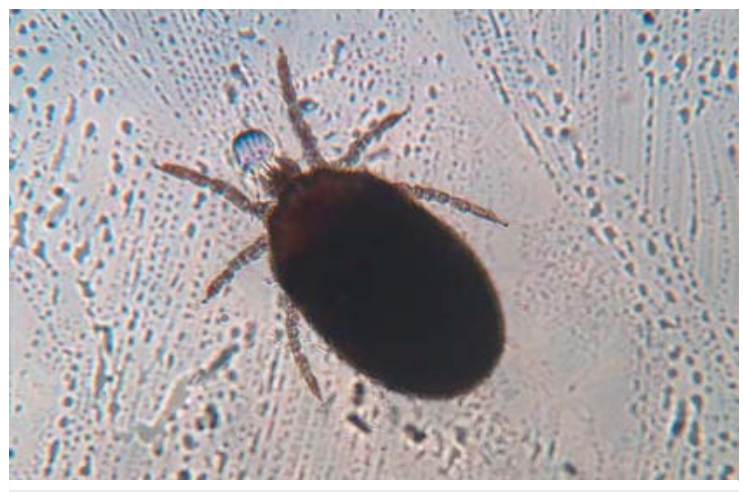

- Abb. 9 Fotomikrograf einer Herbstgrasmilbe aus einem Zwischenzehenbereich einer Katze (100-fache Vergrößerung). Quelle: Ariane Neuber Watts

\section{Kausale Therapie}

Die Allergen-spezifische Immuntherapie (ASIT) stellt die einzige kausale Therapiemöglichkeit dar. Sie kann nach erfolgreicher Diagnosestellung und Ausschluss der Differenzialdiagnosen und anschließendem Allergietest (Intrakutan- oder Serumallergietest, $>$ Abb. 11) aufgrund des Testergebnisses formuliert werden. Dabei wird die Auswahl der Allergene auf die individuelle Symptomatik und die geografische Lokalisation gestützt.

Es gibt verschiedene Formen der Allergen-spezifischen Immuntherapie:

- subkutan (SCIT)

- intralymphatisch (ILIT)

- sublingual (SLIT)

\section{Subkutane Allergen-spezifische Immuntherapie}

Sie wird wohl am häufigsten verwendet und kann mit verschiedenen Protokollen, der klassischen SCIT und der sogenannten „Rush SCIT“ durchgeführt werden. Erstere beinhaltet eine langsame Dosissteigerung über viele Wochen, während beim Rush-Protokoll die volle Dosis innerhalb 1 Tages erreicht wird. Vorteil ist das schnelle Erreichen der maximalen Besserung durch die subkutane Allergen-spezifische Immuntherapie. Bei Hunden wird diese Methode von einigen Dermatologen häufig angewendet. Auch für Katzen gibt es eine Pilotstudie [5], die zu diesem Thema berichtet und bei einer kleinen Gruppe von Katzen diese Behandlung sicher und mit Erfolg angewendet hat. Bei der klassischen Methode dauert es länger, bis die maximale Wirksamkeit erreicht ist, dafür ist sie weniger aufwendig, besser durch Studien belegt und preisgünstiger.

\section{Symptomatische Therapie}

Systemische Therapie

Da der Juckreiz und andere mit allergischer Dermatitis einhergehende Symptome die Lebensqualität des Patienten und der Besitzer stark beeinträchtigen kann, ist die symptomatische Therapie gerade zu Beginn sehr wichtig. Es stehen auch bei der Katze mehrere verschiedene Medikamente zur Auswahl, allerdings sind weniger Wirkstoffe als beim Hund für die Katze zugelassen. Ein paar dieser Präparate sind hauptsächlich als Kortison-sparende Mittel einsetzbar (Antihistaminika, essenzielle Fettsäuren, Palmitoylethanolamide).

\section{Antihistaminika}

Antihistaminika sind eine Art der kausalen Therapie, allerdings sind die meisten verfügbaren Studien sehr alt und waren nicht placebokontrolliert. Eine randomisierte, doppelt-geblindete Kreuzstudie, die die Wirkung von Cetirizin in einer Dosis von $1 \mathrm{mg} / \mathrm{kg}$ p. o. untersuchte, fand keinen Unterschied zwischen Patienten, die Cetirizin oder Placebo erhielten [6]. Zurzeit gibt es kein Antihistaminikum, das für Katzen zugelassen ist und keines, das verlässlich eine gute Wirkung gezeigt hat. Bei einzelnen Katzen kann durch Versuchstherapie mit mehreren verschie- 

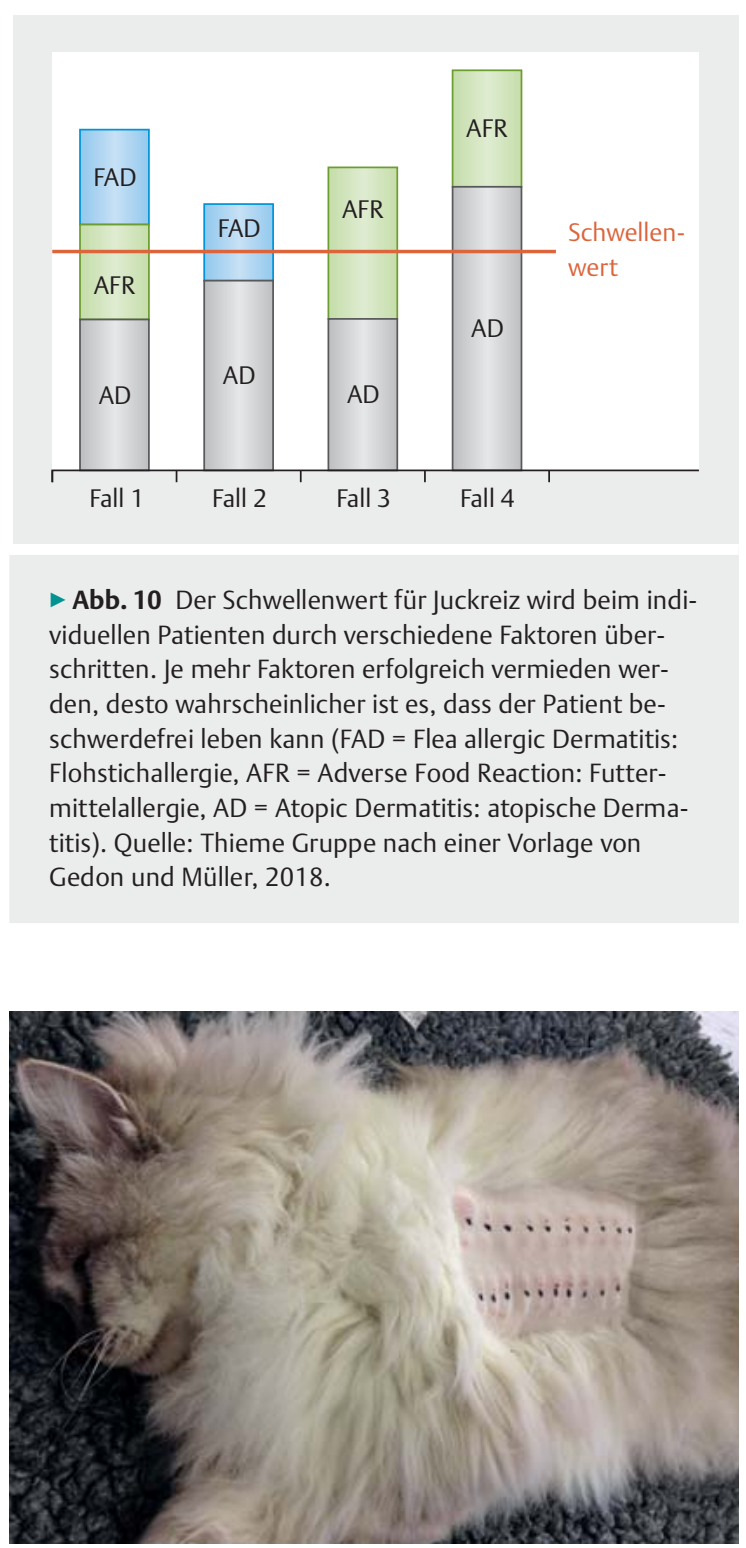

- Abb. 11 Intradermaltest bei einer Katze zur Identifikation der Allergene. Quelle: Ariane Neuber Watts

denen Antihistaminka unter Umständen ein Präparat gefunden werden, das bei diesem Individuum gut wirkt.

\section{Essenzielle Fettsäuren}

Essenzielle Fettsäuren (PUFA: mehrfach ungesättigte Fettsäuren, Omega-3- und Omega-6-Fettsäuren) werden häufig für Hauterkrankungen bei Katzen verschrieben. Auch für diese Wirkstoffe gibt es überwiegend ältere, nicht randomisierte Studien, oft mit unklaren Einschlussbedingungen. Es gibt daher keine schlüssigen Beweise über deren Effizienz. Theoretisch sollten sie die bei allergischen Tieren oft gestörte epidermale Fettschutzschicht verbessern. Vor allem sind sie sicher und können die Hautstruktur allgemein verbessern. 


\section{Palmitoylethanolamide}

Palmitoylethanolamide (PEA) ist ein natürlich vorkommendes Lipid. Es hat entzündungshemmende Eigenschaften, denn es schwächt die Mastzelldegranulation ab. Eine Studie, die dieses Präparat bei Katzen getestet hat [7], fand eine gute Wirkung bei felinen Patienten mit allergischer Dermatitis. Die Studie zeigte eine Verbesserung der Läsionen (Pruritus, Alopezie, eosinophile Granulome, Erythem) bei ca. 2 von 3 der untersuchten Katzen nach Gabe von Palmitoylethanolamide in einer Dosis von $10 \mathrm{mg} / \mathrm{kg}$ p.o. täglich für 30 Tage.

\section{Maropitant}

Eine offene Pilotstudie [8] fand heraus, dass Maropitant bei Katzen gut toleriert wird und effektiv ist, um die nicht Floh-, nicht Futter-induzierte allergische Dermatitis bei der Katze zu behandeln. Maropitant ist ein selektiver Neurokinin-1-Rezeptorinhibitor und wird sonst gegen Erbrechen eingesetzt.

\section{Systemische Glukokortikoide}

Noch vor wenigen Jahren waren Medikamente wie Prednisolon die einzig schnellwirkenden und zuverlässigen Wirkstoffe. Katzen zeigen eine höhere Toleranz gegenüber den unerwünschten Nebenwirkungen der Glukokortikoide, benötigen allerdings eine höhere Dosis. Unerwünschte Nebenwirkungen in diesem Zusammenhang können allerdings dramatisch sein, z. B. kann es zum Diabetes mellitus oder zum sogenannten „skin fragility syndrome“ kommen. Bei dieser Erkrankung können die Glukokortikoide eine Inhibition der Kollagensynthese verursachen. Ansonsten können auch Katzen mit Polydipsie, Polyphagie, Polyurie, Energielosigkeit, erhöhter Anfälligkeit für Infektionen, Erbrechen und Übelkeit reagieren. Diese Nebenwirkungen sind allerdings seltener als bei Hunden. Katzen benötigen oft $1-2 \mathrm{mg} / \mathrm{kg}$ p.o. Prednisolon in der Anfangsphase. Dann wird langsam die Dosis reduziert, bis die niedrigste noch wirksame Dosis gefunden wurde. Auf jeden Fall sollte man versuchen, Prednisolon langfristig nur jeden 2. Tag zu verabreichen.

\section{Ciclosporin}

Ciclosporin (Atopica ${ }^{\circledR}$ ) wird bereits seit vielen Jahren bei Hunden mit caniner atopischer Dermatitis eingesetzt und ist ähnlich effektiv wie Prednisolon. Für Katzen ist es auch seit ein paar Jahren zugelassen und sehr wirksam [9, 10]. Die Dosis ist bei Katzen mit $7 \mathrm{mg} / \mathrm{kg} / \mathrm{Tag}$ p. o. etwas höher als bei Hunden.

Ciclosporin kann bei Katzen starke Inappenz und andere gastrointestinale Symptome auslösen. Besonders bei übergewichtigen Tieren sollte deshalb darauf geachtet werden, dass eine hepatische Lipidose vermieden wird.

\section{PRAXISTIPP}

Eine Toxoplasma-Infektion kann bei Patienten, die mit Ciclosporin behandelt werden, fatal enden. Freigängerkatzen, die noch keinen protektiven Titer gegen Toxoplasmen haben, sollten daher soweit wie möglich vor einer Exposition mit Toxoplasma geschützt werden (Jagen verhindern) [11]. Rohfütterung ist bei Patienten, die mit Ciclosporin behandelt werden, aufgrund der Immunsuppression zu vermeiden. Bei ungeklärten respiratorischen Symptomen ist Ciclosporin sofort abzusetzen. FIV- und FeLV-positive Katzen sollten nicht mit Ciclosporin behandelt werden. Idealerweise wird der Wirkstoff vor und nach der Impfung zwischenzeitlich abgesetzt, damit ein guter Impftiter erreicht werden kann.

\section{Oclacitinib}

Oclacitinib (Apoquel ${ }^{\circledR}$ ) ist nur für Hunde zugelassen. Es handelt sich bei diesem Medikament um einen selektiven Januskinase-1-Inhibitor, der die Signalübertragung bei Zytokinen (z. B. II-2, II-4, II-6, II-13 und II-31) [12] hemmt und den Juckreiz sehr schnell effektiv reduziert. Die Studie von Ortalda et al. [13] zeigte, dass die Therapie mit Oclacitinib auch bei Katzen erfolgreich sein kann, allerdings schlugen sie eine höhere Dosis im Vergleich zum Hund vor. Die Studie von Lopez et al. [14] bestätigte, dass Oclacitinib in einer Dosis von $1 \mathrm{mg} / \mathrm{kg}$ p.o. bei Katzen effektiv und sicher ist. Noli et al. [15] veröffentlichten eine Studie, in der sie Oclacitinib mit Methylprednisolon verglichen. Dabei fanden sie heraus, dass Oclacitinib in einer Dosis von 0,7-1,2 mg/kg p. o. bei Katzen mit nicht Futtermittel-induzierter Hypersensitivitätsdermatitis eine sehr gute Wirkung aufwies, auch wenn Methylprednisolon in einer Dosis von $0,5-1 \mathrm{mg} / \mathrm{kg}$ p.o. etwas effektiver war.

Das Biologikum Lokivetmab, ein caninisierter monoklonaler Antikörper gegen II-31, ist für Katzen aufgrund der Gefahr eines anaphylaktischen Schockes nicht empfohlen. Da II-31 auch bei Katzen in der Pathogenese eine Rolle spielt, wäre dies in Zukunft eventuell ein weiterer Ansatzpunkt für eine systemische Therapie. Bei Hunden wird das Medikament alle 4 Wochen subkutan verabreicht und wirkt bei einer großen Anzahl von Patienten sehr zuverlässig. Für Katzen wäre es aufgrund des selteneren Applikationsintervalls besonders gut geeignet.

\section{Lokale Therapie}

Lokale Therapien sind aufgrund des natürlichen Putzverhaltens der Katze bei vielen Patienten weniger gut geeignet. Sie sollten aber bei lokalen Läsionen trotzdem versucht werden, da sie mit weniger potenziellen Nebenwirkungen verbunden sein können. 
Hydrocortisonaceponat wurde bei einer Katze mit Pemphigus foliaceus erfolgreich angewendet [16]. Schmidt et al. [17] stellten in einer offenen Pilotstudie fest, dass dieser Wirkstoff auch bei Katzen mit allergischer Dermatitis sicher und effizient ist. Allerdings ist das Spray (Cortavance, Virbac) nur für Hunde zugelassen.

Tacrolimus ist ein topisches Präparat aus der Humanmedizin (Protopic), das für Katzen umgewidmet werden kann. Es handelt sich um einen Calcineurin-Hemmer, der bei atopischer Dermatitis angewendet werden kann. Moon et al. [18] haben diesen Wirkstoff gemeinsam mit Prednisolon bei einer Katze mit eosinophilem Granulom erfolgreich angewendet.

\section{Therapie der Verschlimmerungsfaktoren}

Bei der Katze gibt es wie beim Hund Verschlimmerungsfaktoren, die jeden Patienten über die Juckreizschwelle bringen können. Bei Freigängerkatzen ist daher eine umfassende Ektoparasitenkontrolle empfehlenswert. Mögliche Ansatzpunkte sind in diesem Zusammenhang beispielsweise auch Pheromone, die die Ängstlichkeit und dadurch den Stress einer Katze reduzieren sollen. Wildermuth et al. [19] benutzten Amoxicillin-Clavulanat als Monotherapie für feline eosinophile Plaques und Lippenulzera und verglichen die Veränderung mit einer Placebogruppe. Die Katzen, die antibiotisch versorgt wurden, zeigten eine Verkleinerung der Läsionen, die unbehandelten Patienten hingegen eine Vergrößerung. Dies zeigt, dass auch bei Katzen Sekundärinfektionen ein wichtiger Faktor sind und behandelt werden müssen.

\section{Fazit}

Allergische Dermatitiden bei der Katze sind ebenso wie beim Hund ein komplexes Geschehen, die bei vielen Patienten am besten auf eine Kombinationstherapie ansprechen. Die Erkrankung kann nicht geheilt werden, durch ein Managementprogramm kann aber eine gute Lebensqualität erzielt werden.

\section{TAKE HOME}

Allergische Dermatitis bei der Katze:

- multifaktorielle Erkrankung

- Verschlimmerungsfaktoren können zum Überschreiten des Schwellenwerts führen, sodass sich die Symptome verschlechtern können

- empfehlenswert ist die Kombination verschiedener Therapieansätze

- symptomatische Therapie

- zur Behandlung von Juckreiz und sekundären Problemen

- systemische Therapie (Antihistaminika, essenzielle Fettsäuren, Palmitoylethanolamide, Maropitant, systemische Glukokortikoide, Ciclosporin, Oclacitinib, ggf. in Kombination mit Methylprednisolon)

- lokale Therapie (Hydrocortisonaceponat, Tacrolimus)

- kausale Therapie

- Allergen-spezifische Immuntherapie

- Allergenvermeidung

- Therapie der Verschlimmerungsfaktoren

- Verhaltensprobleme/Stress minimieren

- Sekundärinfektionen mit Bakterien oder Hefen therapieren

- Ektoparasitenprophylaxe

Korrespondenzadresse

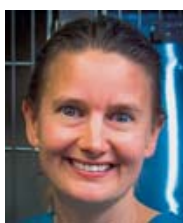

Dr. Ariane Neuber-Watts, CertVD, Dipl. ECVD, MRCVS

Hund Katze Haut

Schulstraße 6

53639 Königswinter

Literatur

Literatur ist in der Online-Version unter www.thieme-connect.de/products einsehbar.

Bibliografie

DOI https://doi.org/10.1055/a-1109-9653

Kleintier konkret 2020; 23: 9-18

(c) Georg Thieme Verlag KG Stuttgart · New York ISSN 1434-9132 